\title{
A!
}

This is an electronic reprint of the original article.

This reprint may differ from the original in pagination and typographic detail.

Karjalainen, Oskari K.; Koskinen, Ari M.P.

\section{Scalable synthesis of (-)-trans-3-hydroxypipecolic acid via a useful chiral building block}

\section{Published in:}

Tetrahedron

DOI:

10.1016/j.tet.2014.02.020

Published: 01/01/2014

Document Version

Peer reviewed version

\section{Please cite the original version:}

Karjalainen, O. K., \& Koskinen, A. M. P. (2014). Scalable synthesis of (-)-trans-3-hydroxypipecolic acid via a useful chiral building block. Tetrahedron, $70(14), 2444-2448$. https://doi.org/10.1016/j.tet.2014.02.020

This material is protected by copyright and other intellectual property rights, and duplication or sale of all or part of any of the repository collections is not permitted, except that material may be duplicated by you for your research use or educational purposes in electronic or print form. You must obtain permission for any other use. Electronic or print copies may not be offered, whether for sale or otherwise to anyone who is not an authorised user. 


\title{
Scalable synthesis of (-)-trans-3-hydroxypipecolic acid via a useful chiral building block
}

\author{
Oskari K. Karjalainen, Ari M.P. Koskinen* \\ Aalto University School of Chemical Technology, Kemistintie 1, 02150 Espoo, Finland
}

\section{A R T I C L E I N F O}

Keywords:

Amino alcohol

Diastereoselective synthesis

Hydroxypipecolic acid

\section{A B S T R A C T}

A scalable synthesis of (-)-trans-2-(hydroxymethyl)-1,2,3,6-tetrahydropyridin3-ol, a versatile chiral building block is described along with its transformation to (-)-trans-3-hydroxypipecolic acid.

(c) 2014 Elsevier Ltd. All rights reserved.

\section{Introduction}

Tetrahydropyridinol $\mathbf{1}$, or its variously protected forms, is a common key building block or intermediate in numerous papers targeting the synthesis of nojirimycin analogs 2 and other iminosugars including sialic acid analogs $\mathbf{3}$ (Fig. 1).,<smiles>OCC1NCC=CC1O</smiles><smiles>C=C</smiles><smiles>CC(C)NC1CNC(CO)C(O)C1O</smiles>

Fig. 1. Uses for the chiral building block 1.

We required an economical, scalable access to $\mathbf{8}$ in enantio- and diastereopure form as part of one of our projects. The project was discontinued sometime after successful delivery of over $10 \mathrm{~g}$ of the target compound (8) in one batch. Due to the continued interest of the synthetic community in derivatives of $\mathbf{1}{ }^{3}$ we wish to convey the full details for the preparation of $\mathbf{8}$. The synthesis is a modified and streamlined version of the synthesis we used to synthesize deoxyaltronojirimycin. ${ }^{4}$

\section{Results and discussion}

The synthesis is outlined in Scheme 1 and begins with Garner's aldehyde $4 .^{5}$ Garner's aldehyde, while expensive to purchase, is readily prepared at moderate scale. ${ }^{6}$ The largest batches prepared were in excess of $130 \mathrm{mmol}$ with $>98 \%$ ee through DIBAL-H reduction of the corresponding methyl ester. Lithium acetylide (1.3 equiv) generated from silyl protected propargyl alcohol was reacted with 4 to give the propargylic alcohol as a mixture of diastereomers (ca. 15:1 anti:syn by ${ }^{1} \mathrm{H}$ NMR spectroscopy) in high yield (typically $>95 \%$ ). This reaction was originally described by Jurczak $^{7}$ and we found that improved anti selectivity is obtained by using THF as the solvent and slowly adding the aldehyde as a precooled solution. The crude product was treated with $\mathrm{NaH}$ in the presence of $\mathrm{BnBr} / \mathrm{NaI}$ in a $\mathrm{DMF} / \mathrm{THF}$ mixture to introduce the benzyl protection. After work up, the reaction mass was filtered through a pad of silica gel to remove any polar impurities/color that might have formed during the previous step. The greasy nature of the compound is highly advantageous as it permits extraction of the product from aqueous DMF mixtures with hydrocarbon solvents, such as hexane and facilitates the silica gel filtration. The silyl protecting group was removed by treatment with ammonium bifluoride in methanol. Ammonium bifluoride is inexpensive and does not generate bothersome tetrabutylammonium impurities associated with TBAF. The crude mass was then loaded on a pad of silica gel and washed with hexane to remove non-polar impurities 
(e.g., excess $\mathrm{BnBr}$ ) to give $\mathbf{5}$ in high purity and yield (91\% crude yield from 4 ) avoiding fractionation by column chromatography.<smiles></smiles><smiles>CC(C)C</smiles><smiles>[R]C/C=C\[C@H](OCc1ccccc1)[C@H]1COC(C)(C)N1C(=O)O</smiles><smiles>CCC#C[C@H](OCc1ccccc1)[C@@H]1COC(C)(C)N1C(C)(C)C</smiles>

Scheme 1. Reagents and conditions: a) $\mathrm{HC} \equiv \mathrm{CCH}_{2} \mathrm{OTBS}, n$-BuLi, THF, $-78{ }^{\circ} \mathrm{C}$, then 4 ; b) $\mathrm{NaH}, \mathrm{BnBr}, \mathrm{NaI}, \mathrm{DMF} / \mathrm{THF}, 0^{\circ} \mathrm{C} ; \mathrm{c}$ ) $\mathrm{NH}_{4} \cdot \mathrm{HF}_{2}, \mathrm{MeOH}$, rt, then silica gel, $91 \%$ over 3 steps; d) Lindlar's catalyst, quinoline, $\mathrm{H}_{2}$, benzene, rt, $98 \%$; e) $\mathrm{POCl}_{3}$, DMF, rt $81 \%$. f) $\mathrm{AcCl}$, $\mathrm{MeOH}$; rt. g) Basic ion exchange resin, $\mathrm{MeOH}$, then cryst from ${ }^{i} \mathrm{PrOH} / \mathrm{EtOH}, 69 \%$ over two steps.

Lindlar reduction was used to introduce the $Z$-double bond into the molecule. ${ }^{8}$ Typically $5 \%$ of the E-double bond was formed as evidenced by ${ }^{1} \mathrm{H}$ NMR spectroscopy, but this is of no consequence as this impurity is completely removed during the final crystallization. High purity 6 was obtained in 98\% crude yield after filtering off the catalyst and acidic washings, thus obviating any need for purification.

A leaving group had to be introduced to the allylic position. We first considered preparing sulfonate esters (mesylate, tosylate) of the primary alcohol. They both worked admirably in the pivotal cyclization, but both gave the same impurity during their formation. The impurity was readily identified as the allyl chloride 7. The chloride possessed improved stability and cleaner reaction profile during the cyclization over the sulfonate esters and was targeted for the synthesis. Initial experiments for the chlorination were conducted with $\mathrm{PCl}_{5}$ and showed promising results on small scale ( $>90 \%$ isolated yield). However, on scaleup, the yields unexplainably dropped to about 50\%. Most likely the traces of chlorine in the reagent were the culprit. Fortunately, $\mathrm{POCl}_{3}$ in DMF proved to be an excellent replacement, providing the allyl chloride in high yield. Interestingly, no reaction took place with 1 equiv of $\mathrm{POCl}_{3}$. With 2 equiv fast formation of an intermediate product was first seen with slower conversion to the chloride. The intermediate was isolated and identified as the formate ester $\mathbf{6}^{\prime}$. Thus, the alcohol is initially formylated with the Vilsmeier reagent produced from $\mathrm{POCl}_{3}$ and DMF (Scheme 2). The formate is then displaced by chloride to give the product and formic acid. We encountered a slight problem during the workup on scale; too rapid addition of $\mathrm{NaOH}$ caused partial hydrolysis of the product back to the allyl alcohol or a related compound. Fortunately, the impurity could be removed during product isolation. The allyl chloride 7 was obtained in $81 \%$ crude yield.

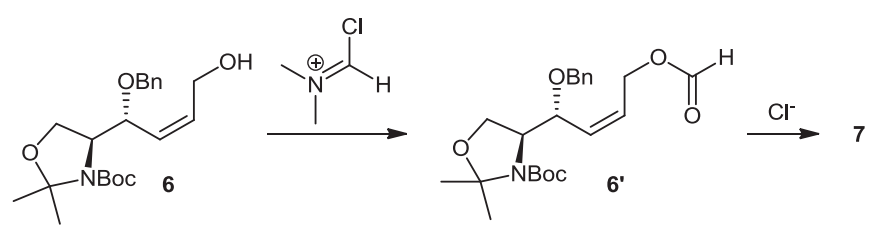

Scheme 2. Chlorination of $\mathbf{6}$ via a formate ester.
The protecting groups were most efficiently removed using methanolic $\mathrm{HCl}$ generated from acetyl chloride. Other acids tested (TFA, TsOH) complicated the product isolation after cyclization, which was accomplished by treating a methanolic solution of the crude acid with basic ion exchange resin at elevated temperature. This enabled the cyclization and after crystallization from ${ }^{i} \mathrm{PrOH} /$ EtOH the product was obtained as a stable, non-hygroscopic hydrochloride salt in $69 \%$ isolated yield. The use of ion exchange resin was highly beneficial as traditional bases (TEA, DIPEA) caused the hydrochloride salt to be contaminated with the salt of the base, requiring multiple crystallizations to upgrade the purity. Use of inorganic bases $\left(\mathrm{K}_{2} \mathrm{CO}_{3}, \mathrm{NaHCO}_{3}\right)$ avoided that problem, but contaminated the crystalline material with inorganics. Otherwise the crystallization is robust, efficiently removing impurities from the upstream chemistry (e.g., the isomeric impurity from the hydrogenation) even at elevated levels.

The utility of $\mathbf{8}$ was demonstrated by transforming it into trans3-hydroxypipecolic acid $\mathbf{1 0}$ (Scheme 3). ${ }^{9}$ We initially attempted to oxidize $\mathbf{8}$ directly to $\mathbf{9}$ under various conditions with little success. Either the reactions did not proceed or more often were messy with plenty of over oxidized compounds detected. On the other hand, the high solubility of $\mathbf{9}$ into aqueous phases limited our choice of oxidants to organic ones. Therefore, compound $\mathbf{8}$ was Boc protected and then subjected to TEMPO catalyzed oxidation with bis(acetoxy) iodobenzene (BAIB) as the terminal oxidant to relatively cleanly give the carboxylic acid. ${ }^{10}$ We found it useful to perform the reaction under biphasic conditions in the presence of sodium bicarbonate. This way the workup was facilitated and the product was partly protected from over oxidation as the sodium carboxylate had very limited solubility in the reaction mixture. After extractive workup the transient Boc-group was cleaved by exposure to $\mathrm{HCl}$ in acetonitrile. The crude $\mathbf{9}$ was efficiently purified by slurrying it in ${ }^{i} \mathrm{PrOH}-$ heptane mixture to give pure $\mathbf{9}$ as a white powder in $63 \%$ yield over three steps. Final hydrogenation delivered $\mathbf{1 0}$ as a colorless crystalline solid entirely without chromatography.<smiles>CC(C)CO</smiles><smiles>O=C(O)[C@H]1NCC=C[C@@H]1Br</smiles><smiles>O=C(O)[C@@H]1NCCC[C@H]1O</smiles>

Scheme 3. Reagents and conditions: a) $\mathrm{Boc}_{2} \mathrm{O}, \mathrm{NEt}_{3}, \mathrm{CH}_{2} \mathrm{Cl}_{2}$, rt; b) cat. TEMPO, BAIB, $\mathrm{NaHCO}_{3}, \mathrm{CH}_{2} \mathrm{Cl}_{2} / \mathrm{H}_{2} \mathrm{O}, 0{ }^{\circ} \mathrm{C}$ to rt; c) $\mathrm{HCl}, \mathrm{MeCN}$, rt to $50{ }^{\circ} \mathrm{C}, 63 \%$ over three steps; d) $\mathrm{Pd} / \mathrm{C}$, $\mathrm{H}_{2}$, EtOH, rt, $97 \%$.

\section{Conclusion}

In conclusion, we have developed a scalable route to tetrahydropyridinol 8 in seven steps from Garner's aldehyde and in 50\% overall yield. The absence of chromatography enables rapid processing ( $<1.5$ weeks by a single person) and the robust crystallization ensures high product quality. These factors, coupled with low-cost reagents, make this route highly desirable for lab-scale synthesis of this intermediate. Furthermore, the intermediate $\mathbf{8}$ was quickly transformed into trans-3-hydroxypipecolic acid in $60 \%$ yield also entirely without chromatography.

\section{Experimental section}

\subsection{General}

Dry dichloromethane and tetrahydrofuran were obtained from a solvent drier (MB SPS-800, neutral alumina). Dimethyl formamide was from a freshly opened bottle. Other solvents used in reactions and in chromatography were of p.a. quality. Reagents were obtained from 
Sigma-Aldrich or from Acros Organics and used as such, unless otherwise stated. TLC monitoring was performed on Merck silica gel $60 \mathrm{~F}_{254}(230-400 \mathrm{mesh}$, aluminum) plates. UV-light $(\lambda=254 \mathrm{~nm})$, and permanganate ( $3 \mathrm{~g} \mathrm{KMnO}_{4}, 20 \mathrm{~g} \mathrm{~K}_{2} \mathrm{CO}_{3}, 5 \mathrm{~mL} 1 \mathrm{M} \mathrm{NaOH}$, diluted to $300 \mathrm{~mL}$ with water) or vanillin (3 g vanillin, $2.5 \mathrm{~mL}$ concd $\mathrm{H}_{2} \mathrm{SO}_{4}$, $1.5 \mathrm{~mL}$ acetic acid, $125 \mathrm{~mL}$ EtOH) stains were used to visualize the plates. Flash chromatography was performed on Merck Silica Gel 60 silica. The Celite used in filtrations was either Fluka Celite 501 or Sigma-Aldrich Celite 535 Coarse. NMR spectra were recorded on Bruker Avance 400 spectrometer. The spectra were calibrated either to TMS ( $\left.{ }^{1} \mathrm{H}: \delta 0.00 \mathrm{ppm}\right), \mathrm{MeOD}\left({ }^{1} \mathrm{H}\right.$ : TMS, $\left.{ }^{13} \mathrm{C} \delta: 49.86 \mathrm{ppm}\right), \mathrm{CDCl}_{3}$ $\left({ }^{13} \mathrm{C}: \delta 77.0 \mathrm{ppm}\right), \mathrm{Cl}_{2} \mathrm{CDCDCl}_{2}\left({ }^{1} \mathrm{H}: \delta 6.00 \mathrm{ppm},{ }^{13} \mathrm{C} \delta: 73.8\right)$, toluene- $d_{8}$ $\left({ }^{1} \mathrm{H}: \delta 2.09 \mathrm{ppm},{ }^{13} \mathrm{C} \delta: 20.4\right)$ or to $\mathrm{D}_{2} \mathrm{O}\left({ }^{1} \mathrm{H}: \delta 4.70 \mathrm{ppm},{ }^{13} \mathrm{C}: \delta 49.5 \mathrm{ppm}\right.$, $\mathrm{MeOH}$ as internal standard) depending on the used solvent. Spectra were recorded at $25^{\circ} \mathrm{C}$, unless otherwise stated. Heating of the NMRsamples was performed using a probe heater. IR spectra were recorded on Perkin-Elmer Spectrum One FTIR machine. Optical rotations were measured with Perkin-Elmer 343 polarimeter using sodium lamp and a $10 \mathrm{~cm}$ quartz cuvette. Melting points were measured with Stuart SMP30 melting point apparatus. HRMS spectra were recorded on Waters Micromass LCT Premier (ESI/TOF) mass spectrometer.

\section{2. (S)-tert-Butyl 4-((R)-1-(benzyloxy)-4-hydroxybut-2-yn-1- yl)-2,2-dimethyloxazolidine-3-carboxylate (5)}

A flame-dried flask under argon was charged with O-TBS propargyl alcohol (23.7 g, $138 \mathrm{mmol}, 130 \mathrm{~mol}-\%)$ and $275 \mathrm{~mL}$ of dry THF. The solution was cooled to $-78{ }^{\circ} \mathrm{C}$ and $n$-BuLi $(57.5 \mathrm{~mL}$, $135 \mathrm{mmol}, 125 \mathrm{~mol} \%, 2.35 \mathrm{M}$ in hexanes) was added over $10 \mathrm{~min}$. The resulting mixture was stirred for an hour. Then $4(22.7 \mathrm{~g}$, $98.8 \mathrm{mmol}, 100 \mathrm{~mol} \%)$ was added as a THF solution $(115 \mathrm{~mL}+20 \mathrm{~mL}$ for washing, precooled to $-78{ }^{\circ} \mathrm{C}$ ) via cannula over $1 \mathrm{~h}$. The resulting solution was stirred for $1 \mathrm{~h}$ and then quenched by adding $100 \mathrm{~mL}$ of satd $\mathrm{NH}_{4} \mathrm{Cl}$. The cooling bath was removed and replaced with a warm water bath. After reaching room temperature, the aqueous layer was extracted with EtOAc $(2 \times 50 \mathrm{~mL})$. The combined organic phases were dried over $\mathrm{Na}_{2} \mathrm{SO}_{4}$ and concentrated to yield $42.3 \mathrm{~g}$ of crude product as slightly yellow oil. An analytical sample was prepared by flash chromatography (10\% EtOAc/hexanes) to afford a colorless oil. $R_{f} 0.56$ (2:1 Hex/EtOAc); $[\alpha]_{D}^{20}-39.6$ (c 2.50, $\mathrm{CH}_{2} \mathrm{Cl}_{2}$ ) lit. $^{7}-40.7$ (c $\left.1.1 \mathrm{CDCl}_{3}\right) ;{ }^{1} \mathrm{H}$ NMR (400 MHz, $\left.\mathrm{C}_{6} \mathrm{D}_{6}, 60{ }^{\circ} \mathrm{C}\right)$ : $\delta 4.64$ (br s, $1 \mathrm{H}), 4.21(\mathrm{~d}, J=2.0,2 \mathrm{H}), 4.00(\mathrm{~m}, 2 \mathrm{H}), 3.72(\mathrm{dd}, J=8.5,7.2$, $1 \mathrm{H}), 1.69(\mathrm{~s}, 3 \mathrm{H}), 1.46(\mathrm{~s}, 3 \mathrm{H}), 1.39(\mathrm{~s}, 9 \mathrm{H}), 0.94(\mathrm{~s}, 9 \mathrm{H}), 0.09(\mathrm{~s}, 6 \mathrm{H})$; ${ }^{13} \mathrm{C} \mathrm{NMR}\left(100 \mathrm{MHz}, \mathrm{CDCl}_{3}, 25{ }^{\circ} \mathrm{C}\right): \delta 154.1,94.9,84.5,52.5,81.3,65.0$, 64.1, 62.5, 51.6, 28.3, 25.7, 25.8 (rotamers), 25.2, 18.2, -5.2; HRMS: calcd for $\mathrm{C}_{20} \mathrm{H}_{37} \mathrm{NO}_{5} \mathrm{Si}+\mathrm{Na} 422.2339$, found 422.2337 .

A flask under argon was charged with the crude product from the previous reaction ( $42.3 \mathrm{~g}$, assumed circa $98.0 \mathrm{mmol}, 100 \mathrm{~mol} \%$ ), and dry DMF (100 mL). The solution was cooled to $0{ }^{\circ} \mathrm{C}$ and benzyl bromide (15.4 mL, $130 \mathrm{mmol}, 130 \mathrm{~mol} \%$ ) was added together with KI (830 mg, $5 \mathrm{mmol}, 5 \mathrm{~mol} \%$ ). Finally sodium hydride (4.8 g, $120 \mathrm{mmol}$, $120 \mathrm{~mol} \%, 60 \%$ dispersion in mineral oil) was added in a single portion. After gas evolution had stopped the slurry was diluted with $100 \mathrm{~mL}$ of dry THF. After $1 \mathrm{~h}$ of stirring, the reaction was quenched by adding $60 \mathrm{~mL}$ of satd $\mathrm{NaHCO}_{3}$ (dropwise at first, until gas no longer forms) and then diluted with $150 \mathrm{~mL}$ of water. The mixture was extracted with EtOAc $(3 \times 100 \mathrm{~mL})$. The combined organic phases were washed with water $(200 \mathrm{~mL})$ and brine, dried over $\mathrm{Na}_{2} \mathrm{SO}_{4}$ and concentrated. The residue was filtered through a pad of silica gel (eluted with $5 \%$ EtOAc/Hexanes) to yield $52.4 \mathrm{~g}$ of light yellow oil. An analytical sample was prepared by flash chromatography (5\% EtOAc/ hexanes) to afford a colorless oil. $R_{f} 0.66$ (30\% EtOAc/Hex); [ $\left.\alpha\right]_{\mathrm{D}}^{20}-76.5$ (c 2.0, $\mathrm{CH}_{2} \mathrm{Cl}_{2}$ ); IR (film): 1705, 1390, 1366, $1089 \mathrm{~cm}^{-1}$; ${ }^{1} \mathrm{H}$ NMR $\left(400 \mathrm{MHz}, \mathrm{CDCl}_{3}\right): \delta 7.21-7.39(\mathrm{~m}, 5 \mathrm{H}), 4.83$ (app. t, $\left.J=11.3 \mathrm{~Hz}, 1 \mathrm{H}\right)$, $4.69-4.74(\mathrm{~m}, 0.5 \mathrm{H}$, rotamers ), 4.51 (dd, $J=12.1,7.2 \mathrm{~Hz}, 1 \mathrm{H}), 4.45-4.48$ (m, 0.5H, rotamers), 4.37 (d, $J=13.4 \mathrm{~Hz}, 2 \mathrm{H}), 4.23-4.29(\mathrm{~m}, 1 \mathrm{H})$,
4.09-4.15 ( $\mathrm{m}, 0.5 \mathrm{H}$ rotamers), 4.01 (app t, $J=8.2 \mathrm{~Hz}, 1 \mathrm{H}$ ), 3.95-3.99 (m, $0.5 \mathrm{H}$, rotamers), $1.66-1.28(\mathrm{~m}, 15 \mathrm{H}), 0.91$ (app d, $J=4.8 \mathrm{~Hz}, 9 \mathrm{H})$, 0.13 (app d, $J=5.5 \mathrm{~Hz}) ;{ }^{13} \mathrm{CNMR}\left(100 \mathrm{MHz}, \mathrm{CDCl}_{3}\right): \delta 152.7,152.0,138.3$, 137.9, 128.9, 128.7, 128.5, 128.3, 128.0, 95.4, 94.8, 81.8, 80.7, 80.3, 71.4, 71.2, 68.3, 67.7, 65.1, 64.7, 61.1, 60.8, 52.2, 28.8, 26.6, 26.3, 26.2, 26.0, 25.6, 24.1, 18.7, -4.6 (ca. 2:1 mixture of rotamers); ${ }^{13} \mathrm{C}$ NMR $\left(\mathrm{Cl}_{2} \mathrm{DCCDCl}_{2}, 90{ }^{\circ} \mathrm{C}\right): \delta 137.9,128.2,128.1,127.7,127.4,94.5,86.2,81.5$, $79.8,71.0,64.2,60.6,53.3,51.5,28.3,25.9,25.7,25.5,18.0,-5.2$ (the carbonyl group resonance is lost at this temperature); HRMS: calcd for $\mathrm{C}_{27} \mathrm{H}_{43} \mathrm{NO}_{5} \mathrm{Si}+\mathrm{Na}$ 512.2832, found 512.282.

The product from the previous reaction $(52.8 \mathrm{~g}$, assumed circa $98 \mathrm{mmol}, 100 \mathrm{~mol} \%$ ) was dissolved in $\mathrm{MeOH}(80 \mathrm{~mL})$ under ambient conditions. $\mathrm{NH}_{4} \cdot \mathrm{HF}_{2}(11.5 \mathrm{~g}, 200 \mathrm{mmol}, 200 \mathrm{~mol} \%$ ) was added and the resulting mixture was stirred for $18 \mathrm{~h}$. To quench the reaction, $30 \mathrm{~g}$ of silica gel was added to the reaction mixture. After $30 \mathrm{~min}$ of stirring, the solution was diluted with $350 \mathrm{~mL}$ of $\mathrm{CH}_{2} \mathrm{Cl}_{2}$, passed through a pad of silica (washed with $10 \% \mathrm{MeOH} / \mathrm{CH}_{2} \mathrm{Cl}_{2}$ ) and concentrated. The crude product was dissolved in 20\% EtOAc/hexanes and loaded onto a pad of silica gel, which was washed with hexanes followed by EtOAc. After concentration a yellow oil was obtained ( $34.0 \mathrm{~g}, 91 \%$ over three steps). An analytical sample was prepared by flash chromatography (25\% EtOAc/hexanes) to afford a colorless oil. $R_{f} 0.47$ (1:1 EtoAc/Hex); $[\alpha]_{\mathrm{D}}^{20}-54.9$ (c 1.0, $\left.\mathrm{CH}_{2} \mathrm{Cl}_{2}\right)$; IR (film): 3436, 2978, 1683, 1392, $1366 \mathrm{~cm}^{-1}$; ${ }^{1} \mathrm{H}$ NMR (400 MHz, $\left.\mathrm{CDCl}_{3}, 50{ }^{\circ} \mathrm{C}\right)$ : $\delta 7.21-7.40(\mathrm{~m}, 5 \mathrm{H}), 4.80(\mathrm{~d}, J=12.1 \mathrm{~Hz} 1 \mathrm{H}), 4.52(\mathrm{~d}, J=12.1 \mathrm{~Hz}, 1 \mathrm{H})$, $4.12-4.36(\mathrm{~m}, 4 \mathrm{H}), 3.93-4.06(\mathrm{~m}, 1 \mathrm{H}), 1.27-1.70(\mathrm{~m}, 15 \mathrm{H}) ;{ }^{13} \mathrm{C} \mathrm{NMR}$ $\left(100 \mathrm{MHz}, \mathrm{CDCl}_{3}\right): \delta 152.5,151.5,137.6,137.3,128.3,128.2,128.0$, 127.8, 127.7, 127.6, 94.9, 94.2, 85.7, 85.4, 82.5, 80.4, 79.9, 70.9, 67.9, 97.8, 64.6, 64.4, 60.4, 60.1, 50.7, 28.3, 26.5, 25.5, 24.8, 23.5 (rotamers); HRMS: calcd for $\mathrm{C}_{21} \mathrm{H}_{29} \mathrm{NO}_{5}+\mathrm{Na} 398.1943$, found 398.1953.

\section{3. (S)-tert-Butyl 4-((R,Z)-1-(benzyloxy)-4-hydroxybut-2-en- 1-yl)-2,2-dimethyloxazolidine-3-carboxylate (6)}

A $500 \mathrm{~mL}$ flask was charged with benzene ( $50 \mathrm{~mL}), \mathrm{Pd} / \mathrm{CaCO}_{3} / \mathrm{Pb}$ (2.54 g, $1.2 \mathrm{mmol}, 1.5 \mathrm{~mol} \%, 5 \%$ in Pd) and quinoline $(3.3 \mathrm{~mL}$, $27.8 \mathrm{mmol}, 35 \mathrm{~mol} \%$ ). The mixture was degassed three times and left to stir under argon for $30 \mathrm{~min}$. Then $\mathbf{5}(29.8 \mathrm{~g}, 79.5 \mathrm{mmol}, 100 \mathrm{~mol} \%)$ was added in a benzene solution $(200 \mathrm{~mL})$ and $\mathrm{H}_{2}$ atmosphere was introduced. The mixture was stirred vigorously for $2.75 \mathrm{~h}$ (rxn complete by NMR), filtered through a pad of Celite and concentrated. The residue was dissolved in EtOAc $(150 \mathrm{~mL})$ and washed with $1 \mathrm{M}$ $\mathrm{HCl}(100 \mathrm{~mL})$ to remove quinoline. The organic layer was dried over $\mathrm{Na}_{2} \mathrm{SO}_{4}$ and concentrated to give the crude product $(29.4 \mathrm{~g}, 98 \%)$ as a slightly yellow oil. An analytical sample was prepared by flash chromatography (20\% EtOAc/hexanes) to afford a colorless oil. $R_{f} 0.43$ (80\% Et $\left.\mathrm{E}_{2} \mathrm{O} / \mathrm{Hex}\right) ;[\alpha]_{\mathrm{D}}^{20}-65.0$ (c 1.00, Et ${ }_{2} \mathrm{O}$ ); IR: 3436, 1698, 1391, $1366 \mathrm{~cm}^{-1}$; ${ }^{1} \mathrm{H}$ NMR $\left(400 \mathrm{MHz}, \mathrm{CDCl}_{3}, \mathrm{rt}\right): \delta 7.30-7.12(\mathrm{~m}, 5 \mathrm{H}), 5.79$ (dddd, $J=0.9,6.1,7.3,11.3 \mathrm{~Hz}, 1 \mathrm{H}), 5.43(\mathrm{t}, J=9.2 \mathrm{~Hz}, 1 \mathrm{H}), 4.52(\mathrm{~d}$, $J=11.7 \mathrm{~Hz}, 1 \mathrm{H}), 4.43(\mathrm{br} \mathrm{s}, 0.5 \mathrm{H}), 4.27(\mathrm{~d}, J=11.7 \mathrm{~Hz}, 1 \mathrm{H}), 4.21-3.96(\mathrm{~m}$, $4 \mathrm{H}), 3.91-3.72(\mathrm{~m}, 2 \mathrm{H}), 2.82$ (br s, $0.5 \mathrm{H}), 1.57-1.23(\mathrm{~m}, 15 \mathrm{H}) ;{ }^{1} \mathrm{H}$ NMR $\left(400 \mathrm{MHz}, \mathrm{Cl}_{2} \mathrm{CDCDCl}_{2}, 80^{\circ} \mathrm{C}\right): \delta 7.40-7.28(\mathrm{~m}, 5 \mathrm{H}), 5.89$ (dddd, $J=1.1$, 6.2, 7.0, $11.3 \mathrm{~Hz}, 1 \mathrm{H}$ ), 5.56 (tdd, $J=1.3,9.5,11.0 \mathrm{~Hz}, 1 \mathrm{H}), 4.63$ (d, $J=12.1 \mathrm{~Hz}, 1 \mathrm{H}), 4.52$ (br s, $1 \mathrm{H}), 4.45(\mathrm{~d}, J=11.7 \mathrm{~Hz}, 1 \mathrm{H}), 4.28-4.12(\mathrm{~m}$, 3H), 4.01-3.91 (m, 2H), $1.60(\mathrm{~s}, 3 \mathrm{H}), 1.53(\mathrm{~s}, 3 \mathrm{H}), 1.50(\mathrm{~s}, 9 \mathrm{H}) ;{ }^{13} \mathrm{C} \mathrm{NMR}$ $\left(100 \mathrm{MHz}, \mathrm{Cl}_{2} \mathrm{CDCDCl}_{2}, 80^{\circ} \mathrm{C}\right): \delta 152.3,138.3,133.2,130.6,128.1,127.6$, 127.4, 94.1, 80.0, 74.1, 70.8, 64.2, 60.5, 58.5, 28.3, 26.6; HRMS: calcd for $\mathrm{C}_{21} \mathrm{H}_{31} \mathrm{NO}_{5}+\mathrm{Na} 400.2100$, found 400.2115 .

\section{4. (S)-tert-Butyl 4-((R,Z)-1-(benzyloxy)-4-chlorobut-2-en-1- yl)-2,2-dimethyloxazolidine-3-carboxylate (7)}

To an ice cooled, stirred solution of 6 (29.4 g, $77 \mathrm{mmol}$, $100 \mathrm{~mol} \%)$ in DMF $(210 \mathrm{~mL})$ was added $\mathrm{POCl}_{3}(15.6 \mathrm{~mL}, 170 \mathrm{mmol}$, $220 \mathrm{~mol} \%)$ under argon. The cooling bath was removed and the solution was stirred overnight. The bright orange reaction was 
quenched by cooling to $0{ }^{\circ} \mathrm{C}$ and slowly adding $2 \mathrm{M} \mathrm{NaOH}$ (ca. $250 \mathrm{~mL}$ ) to a $\mathrm{pH}$ of $>7$. The mixture was extracted with EtOAc $(3 \times 180 \mathrm{~mL})$. The combined organic phases were dried over $\mathrm{Na}_{2} \mathrm{SO}_{4}$ and concentrated. During the neutralization another product had formed, with $R_{f}$ value similar to the starting material. Filtration through a pad silica (eluted with 15\% EtOAc/hexanes) yielded the product $(24.7 \mathrm{~g}, 81 \%$ ) as a colorless oil. No trace of the byproduct could be found. An analytical sample was prepared by flash chromatography (20\% EtOAc/hexanes) to afford a colorless oil. $R_{f} 0.59$ (60\% $\left.\mathrm{Et}_{2} \mathrm{O} / \mathrm{Hex}\right) ;[\alpha]_{\mathrm{D}}^{20}-61.5$ (c 1.00, $\mathrm{CH}_{2} \mathrm{Cl}_{2}$ ); IR (film) 1699, 1384, $1365 \mathrm{~cm}^{-1}$; ${ }^{1} \mathrm{H}$ NMR $\left(400 \mathrm{MHz}, \mathrm{CDCl}_{3}\right): \delta 7.31-7.16(\mathrm{~m}, 5 \mathrm{H})$, $5.88-5.73(\mathrm{~m}, 1 \mathrm{H}), 5.54(\mathrm{q}, J=11.2 \mathrm{~Hz}, 1 \mathrm{H}), 4.54(\mathrm{dd}, J=8.1,11.7 \mathrm{~Hz}$, $1 \mathrm{H}), 4.40-4.32(\mathrm{~m}, 0.5 \mathrm{H}), 4.29(\mathrm{~d}, J=11.7 \mathrm{~Hz}, 1 \mathrm{H}), 4.26-4.19(\mathrm{~m}$, $0.5 \mathrm{H}), 4.13-3.73(\mathrm{~m}, 5 \mathrm{H}), 1.57-1.28(\mathrm{~m}, 16 \mathrm{H}) ;{ }^{13} \mathrm{C}$ NMR $(100 \mathrm{MHz}$, $\left.\mathrm{CDCl}_{3}\right): \delta 152.6,151.9,137.9,137.6,132.5,130.2,129.8,128.5,128.3$, 128.0, 127.9, 127.6, 94.5, 93.8, 80.1, 73.3, 70.8, 64.8, 64.2, 60.4, 59.8, 39.9, 38.9, 28.4, 28.3, 27.1, 26.6, 24.6, 23.0 (rotamers); ${ }^{1} \mathrm{H}$ NMR (400 MHz, $\left.\mathrm{Cl}_{2} \mathrm{CDCDCl}_{2}, 90{ }^{\circ} \mathrm{C}\right): \delta 7.41-7.29(\mathrm{~m}, 5 \mathrm{H}), 5.94-5.86(\mathrm{~m}$, $1 \mathrm{H}), 5.65(\mathrm{dd}, J=9.5,10.6 \mathrm{~Hz}, 1 \mathrm{H}), 4.65(\mathrm{~d}, J=11.7 \mathrm{~Hz}, 1 \mathrm{H}), 4.54-4.48$ $(\mathrm{m}, 1 \mathrm{H}), 4.45(\mathrm{~d}, J=12.1 \mathrm{~Hz}, 1 \mathrm{H}), 4.21(\mathrm{dd}, J=8.4,11.3 \mathrm{~Hz}, 1 \mathrm{H}), 4.16(\mathrm{~d}$, $J=6.6 \mathrm{~Hz}, 1 \mathrm{H}), 4.11(\mathrm{dd}, J=7.3,12.1 \mathrm{~Hz}, 1 \mathrm{H}), 3.96(\mathrm{td}, J=6.2,6.3 \mathrm{~Hz}$, $1 \mathrm{H}), 4.01-3.93(\mathrm{~m}, 1 \mathrm{H}), 1.61(\mathrm{~s}, 3 \mathrm{H}), 1.55(\mathrm{~s}, 3 \mathrm{H}), 1.51(\mathrm{~s}, 9 \mathrm{H}) ;{ }^{13} \mathrm{C}$ NMR (100 MHz, $\left.\mathrm{Cl}_{2} \mathrm{CDCDCl}_{2}, 90{ }^{\circ} \mathrm{C}\right): \delta 138.1,132.5,129.6,128.2$, 127.7, 127.5, 94.1, 79.9, 73.9, 71.0, 64.3, 60.3, 39.2, 28.3, 26.7; HRMS calcd for $\mathrm{C}_{21} \mathrm{H}_{30} \mathrm{NO}_{4} \mathrm{Cl}+\mathrm{Na} 418.1761$, found 418.1736 .

\section{5. ((2S,3R)-3-(Benzyloxy)-1,2,3,6-tetrahydropyridin-2-yl) methanol hydrochloride $(8)$}

Acetyl chloride (30 mL, $420 \mathrm{mmol}$ ) was added to ice cooled methanol $(90 \mathrm{~mL})$ under argon over $10 \mathrm{~min}$. The solution was stirred for $30 \mathrm{~min}$ and then poured over neat $7(24.7 \mathrm{~g}, 62.0 \mathrm{mmol}$, $100 \mathrm{~mol}-\%)$. After $30 \mathrm{~min}$ of stirring at room temperature, the starting material was completely consumed according to TLC. The solvent was evaporated in vacuo to yield $18.9 \mathrm{~g}$ of crude product as a blood red/brown glassy substance.

The crude product was dissolved in methanol. Then ion exchange resin (Merck ionenaustauscher II; weakly basic tertiary amine resin, $20 \mathrm{~g}, 5 \mathrm{meq} / \mathrm{g}$, moist, ca. $200 \mathrm{~mol} \%$ ) was added and the mixture was vigorously stirred until the solution became neutral. Then the mixture was filtered through a sintered glass funnel and concentrated. The residue was dissolved in $15 \mathrm{~mL} i-\mathrm{PrOH}$ and $15 \mathrm{~mL} \mathrm{EtOH}$ and stirred for $16 \mathrm{~h}$ at $75^{\circ} \mathrm{C}$. Upon cooling $3.4 \mathrm{~g}$ of fine microcrystalline powder was obtained. The mother liquor was concentrated and dissolved in methanol. Another $20 \mathrm{~g}$ batch of the resin was added to neutralize the $\mathrm{pH}$. The mixture was heated to reflux and after $2 \mathrm{~h}$ filtered through a sintered glass funnel and concentrated. The now solid crude was crystallized from $i$-PrOH/EtOH to yield $6.7 \mathrm{~g}$ of medium sized needles. A second crop yielded $840 \mathrm{mg}$ of the said needles for a total of $10.94 \mathrm{~g}\left(69 \%, 50 \%\right.$ over seven steps). $R_{f} 0.66(10 \% \mathrm{MeOH} /$ $\mathrm{CH}_{2} \mathrm{Cl}_{2}+1 \% 25 \%$ aqueous ammonia); mp: $196^{\circ} \mathrm{C} ;[\alpha]_{\mathrm{D}}^{20}-109.1$ (c 1.00, $\mathrm{MeOH})$; IR (KBr disc) 3320, 1585, $1087 \mathrm{~cm}^{-1}$; ${ }^{1} \mathrm{H}$ NMR $(400 \mathrm{MHz}$, $\mathrm{CD}_{3} \mathrm{OD}$ ): $\delta$ 7.40-7.26 (m, 5H), 6.14 (dddd, $J=1.1,7.4,8.7,11.0 \mathrm{~Hz}, 1 \mathrm{H}$ ), $5.96-5.89(\mathrm{~m}, 1 \mathrm{H}), 4.71(\mathrm{~d}, J=11.5 \mathrm{~Hz}, 1 \mathrm{H}), 4.63(\mathrm{~d}, J=11.5 \mathrm{~Hz}, 1 \mathrm{H})$, $4.24-4.18(\mathrm{~m}, 1 \mathrm{H}), 3.87$ (dd, $J=11.9,3.8 \mathrm{~Hz}, 1 \mathrm{H}), 3.79-3.70(\mathrm{~m}, 2 \mathrm{H})$, 4.23 (ddt, $J=17.4,3.5,1.8 \mathrm{~Hz}, 1 \mathrm{H}), 3.42(\mathrm{dt}, J=6.7,3.8 \mathrm{~Hz}, 1 \mathrm{H}) ;{ }^{13} \mathrm{C} \mathrm{NMR}$ (100 MHz, CD $\left.{ }_{3} \mathrm{OD}\right): \delta 139.9,130.3,130.0,129.9,128.4,124.5,73.3,70.4$, 59.4, 59.3, 42.8; HRMS: calcd for $\mathrm{C}_{13} \mathrm{H}_{17} \mathrm{NO}_{2}+\mathrm{H} 220.1338$, found 220.1340; Anal. Calcd for $\mathrm{C}_{13} \mathrm{H}_{18} \mathrm{ClNO}_{2}$ : C, 61.05; H, 7.09; N, 5.48; found: C, 61.22; H, 6.84; N, 5.46.

\section{6. (2R,3R)-3-(Benzyloxy)-1,2,3,6-tetrahydropyridine-2- carboxylic acid hydrochloride (9)}

To a stirred mixture of $\mathbf{8}(2.00 \mathrm{~g}, 8.85 \mathrm{mmol}, 100 \mathrm{~mol} \%)$ in $\mathrm{CH}_{2} \mathrm{Cl}_{2}$ $(20 \mathrm{~mL})$ was added triethylamine $(2.78 \mathrm{~mL}, 19.9 \mathrm{mmol}, 225 \mathrm{~mol} \%)$ followed by $\mathrm{Boc}_{2} \mathrm{O}$ (2.42 g, $\left.11.1 \mathrm{mmol}, 125 \mathrm{~mol} \%\right)$. The mixture was stirred for $2 \mathrm{~h}$ and then poured into $1 \mathrm{~N} \mathrm{HCl}(20 \mathrm{~mL})$. The organic phase was washed with $1 \mathrm{~N} \mathrm{HCl}(20 \mathrm{~mL})$ and the combined aqueous phases were back extracted with $\mathrm{CH}_{2} \mathrm{Cl}_{2}(1 \times 20 \mathrm{~mL})$. The combined organic phases were dried over $\mathrm{Na}_{2} \mathrm{SO}_{4}$ and concentrated to give $3.42 \mathrm{~g}$ of crude as a light yellow oil.

The crude (assumed $8.85 \mathrm{mmol}, 100 \mathrm{~mol} \%$ ) was dissolved in $\mathrm{CH}_{2} \mathrm{Cl}_{2}(30 \mathrm{~mL}) . \mathrm{NaHCO}_{3}(1.50 \mathrm{~g}, 17.7 \mathrm{mmol}, 200 \mathrm{~mol} \%$ ) was added as a solution in water $(30 \mathrm{~mL})$. The resulting vigorously stirred biphasic mixture was cooled to $0{ }^{\circ} \mathrm{C}$ and TEMPO $(275 \mathrm{mg}, 1.77 \mathrm{mmol}$, $20 \mathrm{~mol} \%$ ) was added followed by BAIB (6.27 g, $19.5 \mathrm{mmol}$, $220 \mathrm{~mol} \%$ ). After circa $20 \mathrm{~min}$ of reaction a thick slurry had formed, which is most likely the sodium salt of the product. The mixture was stirred for $16 \mathrm{~h}$ and then quenched with $10 \%$ aqueous $\mathrm{Na}_{2} \mathrm{~S}_{2} \mathrm{O}_{3}$ $(8 \mathrm{~mL}) .2 \mathrm{~N} \mathrm{KOH}(20 \mathrm{~mL})$ was added and the phases were separated. The organic phase was diluted with MTBE $(30 \mathrm{~mL})$ and extracted with $2 \mathrm{~N} \mathrm{KOH}(1 \times 20 \mathrm{~mL})$. The combined aqueous phases were washed with MTBE $(1 \times 30 \mathrm{~mL})$ and carefully acidified with $3 \mathrm{~N} \mathrm{HCl}$ at $0{ }^{\circ} \mathrm{C}$. The turbid mixture was extracted with MTBE $(3 \times 35 \mathrm{~mL})$ and the combined organic phases were dried over $\mathrm{Na}_{2} \mathrm{SO}_{4}$ and concentrated to give $2.17 \mathrm{~g}$ of the crude acid as slightly yellow oil.

The crude product ( $2.17 \mathrm{~g}$, assumed $6.5 \mathrm{mmol}, 100 \mathrm{~mol} \%$ ) was dissolved in $\mathrm{MeCN}(15 \mathrm{~mL})$ and then $\mathrm{HCl}(2.3 \mathrm{~mL}, 22.8 \mathrm{mmol}, 32 \%$ aq) was added at $0{ }^{\circ} \mathrm{C}$. The solution was allowed to warm to rt and then gently heated to $50{ }^{\circ} \mathrm{C}$ for $2 \mathrm{~h}$ to finish the deprotection. The mixture was concentrated to a cream colored solid. The solid was slurried in a mixture of $i-\mathrm{PrOH}$ and heptane $(1: 2,10 \mathrm{~mL})$ at $50{ }^{\circ} \mathrm{C}$ for $2 \mathrm{~h}$, then cooled to $\mathrm{rt}$ and placed in an ice bath to finalize the precipitation. The product was isolated by filtration and the filter cake was washed with $50 \%$ EtOAc/heptane to give $\mathbf{9}$ (1.52 g, 63\% over three steps) as a white powder. Mp 187-189 ${ }^{\circ} \mathrm{C}$ (decomp.); $[\alpha]_{\mathrm{D}}^{20}-105.8$ (c 1.00, MeOH); IR (KBr disc) 2965, 1743, 1567, 1440, $1420,1211 \mathrm{~cm}^{-1} ;{ }^{1} \mathrm{H}$ NMR (400 MHz, $\left.\mathrm{D}_{2} \mathrm{O}\right): \delta 7.51-7.40(\mathrm{~m}, 5 \mathrm{H}), 6.11$ (ddt, $J=10.5,4.5,2.2 \mathrm{~Hz}, 1 \mathrm{H}$ ), 6.01 (dt, $J=10.6,2.9 \mathrm{~Hz}, 1 \mathrm{H}$ ), 4.77 (d, $J=11.3 \mathrm{~Hz}, 1 \mathrm{H}), 4.73(\mathrm{~d}, J=11.3 \mathrm{~Hz}, 1 \mathrm{H}), 4.58(\mathrm{t}, J=4.0 \mathrm{~Hz}, 1 \mathrm{H}), 4.40(\mathrm{~d}$, $J=4.0 \mathrm{~Hz}, 1 \mathrm{H}$ ), 3.97 (ddd, $J=17.7,4.3,2.1 \mathrm{~Hz}, 1 \mathrm{H}), 3.81-3.72(\mathrm{~m}, 1 \mathrm{H})$; ${ }^{1} \mathrm{H}$ NMR (400 MHz, CD ${ }_{3} \mathrm{OD}$ ): $\delta$ 7.45-7.28 (m, 5H), 6.11 (ddt, $J=10.6$, 4.6, $2.3 \mathrm{~Hz}, 1 \mathrm{H}), 5.99$ (dt, $J=10.6,3.1 \mathrm{~Hz}, 1 \mathrm{H}), 4.74(\mathrm{~d}, J=11.7 \mathrm{~Hz}, 1 \mathrm{H})$, $4.69(\mathrm{~d}, J=11.7 \mathrm{~Hz}, 1 \mathrm{H}), 4.55(\mathrm{~d}, J=3.5 \mathrm{~Hz}, 1 \mathrm{H}), 4.46(\mathrm{t}, J=4.1 \mathrm{~Hz}, 1 \mathrm{H})$, 3.96 (ddd, $J=17.7,4.0,2.4 \mathrm{~Hz}, 1 \mathrm{H}$ ), 3.72 (dddd, $J=17.7,3.3,2.4,1.1$, $1 \mathrm{H}) ;{ }^{13} \mathrm{C}$ NMR (100 MHz, CD $\left.3 \mathrm{OD}\right): \delta 168.5,138.8,129.5,129.3,129.1$, 125.5, 125.3, 72.5, 69.4, 57.3, 41.2; HRMS calcd for $\mathrm{C}_{13} \mathrm{H}_{15} \mathrm{NO}_{3}+\mathrm{H}$ 234.1330 , found 234.1131 .

\section{7. $(2 R, 3 R)$-3-Hydroxypiperidine-2-carboxylic acid (10)}

To a solution of $\mathbf{9}(1.0 \mathrm{~g}, 3.7 \mathrm{mmol}, 100 \mathrm{~mol} \%)$ in $\mathrm{MeOH}(15 \mathrm{~mL})$ was added Pd/C (200 mg, $0.19 \mathrm{mmol}, 5 \mathrm{~mol} \%, 10 \mathrm{w} \% \mathrm{Pd}$ ) after which the solution was vacuum degassed followed by introduction of $\mathrm{H}_{2}$ atmosphere. The mixture was stirred under $\mathrm{H}_{2}$ for $16 \mathrm{~h}$ and then filtered through a pad of Celite and concentrated to give $652 \mathrm{mg}(97 \%)$ of yellowish partly crystalline solid. The purity was upgraded by suspending the solids in $\mathrm{EtOH} / \mathrm{CHCl}_{3}(1: 3,5 \mathrm{~mL})$ at $75^{\circ} \mathrm{C}$ for $1 \mathrm{~h}$. Then the mixture was cooled to room temperature and filtered. The filter cake was washed with cold $\mathrm{EtOH} / \mathrm{CHCl}_{3}(1: 3)$ and dried to give $\mathbf{1 0}$ (635 $\mathrm{mg}, 95 \%)$ as a white microcrystalline powder with a hint of rosy color. Mp: $178-181{ }^{\circ} \mathrm{C}$ (decomp.); $[\alpha]_{\mathrm{D}}^{20}$ -14.6 (c 1.10, $\mathrm{H}_{2} \mathrm{O}$ ), lit. for the enantiomer: $+14.2\left(c \quad 0.95, \mathrm{H}_{2} \mathrm{O}\right),{ }^{9 \mathrm{f}}$ +14.5 (c 0.4, $\mathrm{H}_{2} \mathrm{O}$ ) ${ }^{9 \mathrm{e}} \mathrm{IR}$ ( $\mathrm{KBr}$ disc) 3173, 2981, 1744, 1404, 1280, $1081 \mathrm{~cm}^{-1}$; ${ }^{1} \mathrm{H}$ NMR $\left(400 \mathrm{MHz}, \mathrm{CD}_{3} \mathrm{OD}\right.$ ): $\delta 4.17$ (ddd, $J=6.4,6.4$, $3.1 \mathrm{~Hz}, 1 \mathrm{H}), 3.91(\mathrm{~d}, J=6.4 \mathrm{~Hz}, 1 \mathrm{H}), 3.37-3.32(\mathrm{~m}, 1 \mathrm{H}), 3.16-3.06(\mathrm{~m}$, $1 \mathrm{H}), 3.15-3.06(\mathrm{~m}, 1 \mathrm{H}), 2.15-2.03(\mathrm{~m}, 1 \mathrm{H}), 1.92-1.81(\mathrm{~m}, 1 \mathrm{H})$, 1.80-1.65 (m, 2H); ${ }^{13} \mathrm{C}$ NMR (100 MHz, CD $30 D$ ): $\delta 67.0,62.9,44.2$, 30.4, 20.1; (The carbonyl carbon was not visible when run in MeOD); ${ }^{1} \mathrm{H}$ NMR (400 MHz, $\mathrm{D}_{2} \mathrm{O}$ ): $\delta 4.07$ (dt, $\left.J=8.1,2.9 \mathrm{~Hz}, 1 \mathrm{H}\right), 3.75$ (d, $J=7.9 \mathrm{~Hz}, 1 \mathrm{H}$ ), 3.31 (ddd, $J=12.8,6.6,3.8 \mathrm{~Hz}, 1 \mathrm{H}$ ), 4.40 (ddd, 
$J=12.7,9.2,3.1 \mathrm{~Hz}, 1 \mathrm{H}), 2.01-1.90(\mathrm{~m}, 2 \mathrm{H}), 1.74-1.56(\mathrm{~m}, 2 \mathrm{H}) ;{ }^{13} \mathrm{C}$ NMR (100 MHz, $\left.\mathrm{D}_{2} \mathrm{O}\right): \delta 170.5,66.0,61.4,43.0,29.2,19.1$; HRMS calcd for $\mathrm{C}_{6} \mathrm{H}_{12} \mathrm{NO}_{3}+\mathrm{H}$ 146.0817, found 146.0815 .

\section{Acknowledgements}

Funding from the National Graduate School of Organic Chemistry and Chemical Biology (to O.K.K.) is gratefully acknowledged.

\section{Supplementary data}

Supplementary data related to this article can be found at http:// dx.doi.org/10.1016/j.tet.2014.02.020.

\section{References and notes}

1. Compain, P.; Martin, O. R. Iminosugars-From Synthesis to Therapeutic Applications; John Wiley \& Sons Ltd: West Sussex England, 2007.

2. Al-Rawi, S.; Hinderlich, S.; Reutter, W.; Giannis, A. Angew. Chem., Int. Ed. 2004, 43, 4366-4370.

3. For examples see: (a) Shirai, M.; Okamoto, S.; Sato, F. Tetrahedron Lett. 1999, 40, 5331-5332; (b) Martin, R.; Moyano, A.; Pericás, M. S.; Riera, A. Org. Lett. 2000, 2 93-95; (c) Singh, O. V.; Han, H. Tetrahedron Lett. 2003, 44, 2387-2391; (d) Montoro, R.; Márquez, F.; Llebaria, A.; Delgado, A. Eur. J. Org. Chem. 2003, 217-223; (e) Kato, A.; Kato, N.; Kano, E.; Adachi, I.; Ikeda, K.; Yu, L.; Okamoto, T.; Banba, Y.; Ouchi, H.; Takahata, H.; Asano, N. J. Med. Chem. 2005, 48 2036-2044; (f) Martin, R.; Murruzzu, C.; Pericás, M. A.; Riera, A. J. Org. Chem. 2005, 70, 2325-2328; (g) Pyun, S.-J.; Lee, K.-Y.; Oh, C.-Y.; Joo, J.-E.; Cheon, S.-H.; Ham, W.-H. Tetrahedron 2005, 61, 1413-1416; (h) Guaragna, A.; D'Errico, S. D'Alonzo, D.; Pedatella, S.; Palumbo, G. Org. Lett. 2007, 9, 3473-3476; (i)
Guaragna, A.; D’Alonzo, D.; Paolella, C.; Palumbo, G. Tetrahedron Lett. 2009, 50, 2045-2047; (j) Ferreira, F.; Botuha, C.; Chemla, F.; Pérez-Luna, A. J. Org. Chem. 2009, 74, 2238-2241; (k) Rengasamy, R.; Curtis-Long, M. J.; Pyu, H. W.; Oh, K. Y.; Park, K. H. Bull. Korean Chem. Soc. 2009, 30, 1351-1354; (1) Singh, A.; Kim, B. Lee, W. K.; Ha, H.-J. Org. Biomol. Chem. 2011, 9, 1372-1380; (m) van den Berg, R. J. B. H. N.; Wennekes, T.; Ghisaidoobe, A.; Donker-Koopman, W. E.; Strijland, A.; Boot, R. G.; van der Marel, G. A.; Aerts, J. M. F. G.; Overkleeft, H. S. ACS Med. Chem. Lett. 2011, 2, 519-522; (n) Chattopadhyay, S. K.; Roy, S. P.; Saha, T. Synthesis 2011, 2664-2670; (o) Jarvis, S. B. D.; Charette, A. B. Org. Lett. 2011, 13 , 3830-3833; (p) Kalamkar, N. B.; Dhavale, D. D. Tetrahedron Lett. 2011, 52, 6363-6365; (q) Ramalingam, S.; Bhise, A. D.; Show, K.; Kumar, P. ARKIVOC 2013 ii, 220-227; (r) de la Fuente, A.; Martin, R.; Mena-Barragán, T.; Verdaguer, X. Garcia Fernández, J. M.; Ortiz Mellet, C.; Riera, A. Org. Lett. 2013, 15, 3638-3641.

4. Karjalainen, O. K.; Koskinen, A. M. P. Org. Biomol. Chem. 2011, 9, 1231-1236.

5. (a) Liang, X.; Andersch, J.; Bols, M. J. Chem. Soc., Perkin Trans. 1 2001, 2136-2157; (b) Reginato, G.; Meffre, P.; Gaggini, F. Amino Acids 2005, 29, 81-87; (c) Passiniemi, M.; Koskinen, A. M. P. Beilstein J. Org. Chem. 2013, 9, 2641-2659.

6. For reductive approach see: Koskinen, A. M. P.; Hassila, H.; Myllymäki, V. T.; Rissanen, K. Tetrahedron Lett. 1995, 36, 5619-5622 For oxidative approach see: Dondoni, A.; Perrone, D. Org. Synth. 2000, 77, 64-73; Org. Synth. 2004; Collect. Vol. 10, 320-326.

7. Gruza, H.; Kiciak, K.; Krasiński, A.; Jurczak, J. Tetrahedron: Asymmetry 1997, 8 $2627-2631$.

8. Lindlar, H.; Dubuis, R. Org. Synth. 1966, 46, 89-92 Org. Synth. 1973; Collect. Vol. 5, 880-888.

9. For recent reviews see: (a) Cant, A. A.; Sutherland, A. Synthesis 2012, 44 1935-1950; (b) Cochi, A.; Pardo, D. G.; Cossy, J. Eur J. Org. Chem. 2013, 809-829 For selected recent syntheses, see: (c) Liang, N.; Datta, A. J. Org. Chem. 2005, 70 10182-10185; (d) Wang, B.; Liu, R.-H. Eur. J. Org. Chem. 2009, 2845-2851; (e) Chiou, W.-H.; Lin, G.-H.; Liang, C.-W. J. Org. Chem. 2010, 75, 1748-1751; (f) Lemire, A.; Charette, A. B. J. Org. Chem. 2010, 75, 2077-2080; (g) Huy, P. H.; Koskinen, A. M. P. Org. Lett. 2013, 15, 5178-5181.

10. Epp, J. B.; Widlanski, T. S. J. Org. Chem. 1999, 64, 293-295. 